\title{
CAN-BUS APPLICATION SYSTEM IN CLOSE LOOP CONTROL SYSTEMS FOR HEAVY MACHINES
}

\author{
Michał Bednarski \\ Institute of Cranes and Heavy Machinery \\ Engineering, Warsaw University of Technology \\ 84 Narbutta St. 02-524 Warsaw, Poland \\ mibedn@o2.pl
}

\author{
Dariusz Dąbrowski \\ Institute of Cranes and Heavy Machinery \\ Engineering, Warsaw University of Technology \\ 84 Narbutta St. 02-524 Warsaw, Poland \\ Dariusz.Dabrowski@simr.pw.edu.pl
}

\author{
Jan Szlagowski \\ Institute of Cranes and Heavy Machinery Engineering, Warsaw University of Technology \\ 84 Narbutta St. 02-524 Warsaw, Poland \\ Jan.Szlagowski@simr.pw.edu.pl
}

\begin{abstract}
This paper describes the examples of practical applications of CAN-bus system in close control loop system of the equipment for the heavy machines. The document covers three topics. The first one is using CAN-bus in control systems, its pros (small cable harness, easier servicing, etc.) and cons (such as asynchronous data transfer, availability of drivers). The document describes how design of network may minimize impact of cons. There is simulator focused on data acquisition system parameters (such as resolution, asynchronous factor, control loop time) described in the second topic. The role of simulator is to estimate minimal requirements of data acquisition system to achieve desired precision of results while using specified control algorithm. The last topic presents an idea of control algorithm based on stabilization of velocity vector. The aim of that algorithm is to realize trajectory in situation when very exact coordination of hydraulic cylinder rods is needed.
\end{abstract}

\section{KEYWORDS}

Automation systems, Can-bus, digital control of the hydraulic systems

\section{USING CAN-BUS [1], [2]}

Heavy machines controlled by computers need to use a lot of signals. Signals are used for transferring data from gauges to computer (like positions of cylinder rods, their actual velocity, pressure in important points of equipment, etc.) and from computer to valves or engines. In systems where analogue gauges are in use every element of system needs to be connected by at least signal one cable (assuming that GND is connected to chassis) or a pair of cables. Additionally power has to be supplied to gauges. This 
leads to first inconvenience which is cable harness. E.g. at system of typical excavator there are measured four positions and eight pressures, so it needs twelve signal cable pairs and the same number of power cables. This estimation contains only parts used for digging (bucket, arm, beam, rotation). Why cable harness is a problem? Because it enlarge maintenance costs and time. Finding a defect and replacing or servicing broken cable in whole bunch is more difficult and time consuming.

Another issue is connected with type of signal. When gauge has analogue output a signal received by computer contains noise as well. One never know how much noise is in signal one have received. It is possible to calculate average of last several measurements but it is still an inconvenience and inserts an error to further calculations.

The solution for above issues might be using digital bus for transferring data. The network which has been chosen in Institute of Cranes and Heavy Machinery laboratory is CAN-bus. It has been chosen because of simplicity of cabling and fact it was designed (and is used) for wide range of mobile applications: cars, buses, trains, forestry and agricultural machinery. Another benefit of CAN-bus (and other digital buses) is that received data are always true. In case of noise and damage of data or other communication problems data frame is retransmitted. The case computer did not received data is the indicator of broken transmission (broken cable or gauge) and proper decision may be taken immediately.

Despite benefits CAN-bus has issues as well. First one is availability of drivers. At early stage of test stand design we were faced the fact that purchased CAN-bus cards had drivers only for Windows and Linux and neither worked correctly.

Another feature that may bring doubts about using CAN-bus in control systems is asynchronous data transfer. The fact that nodes are connected to the same wire cause that data cannot be transferred in the same time. Moreover process of conversion of physical value into data in every gauge is started independently so measurements have origins in different time points. By using technical specification we can estimate maximum age (difference between time when physical value was captured and time when data frame was transmitted) of received data. E.g. transducers manufactured by Balluff series BTL [3] are able to send data frames every millisecond so we can deduct that time of conversion is lower than 1 millisecond.

In basic model of CAN-bus usage data frames are transmitted by every node periodically with constant period (time between consequent frames). The time of frame is also known and depends on data section length (according to CAN specification data section is limited to 8 bytes). E.g. for bit rate of $250 \mathrm{kbps}$ one full-length frame takes about 500 microseconds. This allows to design network properly. Let's assume that one have 4 transducers (transmit position and velocity) and 8 pressure gauges. Total time of sending frames by all gauges is $(4+8) * 500$ $[\mu \mathrm{s}]=6[\mathrm{~ms}]$. This means that is if we set up sending period of all gauges for more than 6 milliseconds all gauges will be able to send its data frames. This means also that control loop period should be longer than 6 milliseconds. Otherwise control algorithm will not have fresh data in every cycle. We also should remember that values of measurements have origins in different time points. This leads to dissynchronization of measurements and control loop calculations. We can define asynchronous factor for cyclic transmission as follows (worst case):

$k_{a c}=\frac{t_{a}+t_{f}+T_{g}}{T_{c}}$

where:

$\mathrm{k}_{\mathrm{ac}}$ - asynchronous factor for cyclic transmission

$t_{a}$ - age of transmitted data

$t_{f}$ - time of frame

$\mathrm{T}_{\mathrm{g}}$ - transmission period

$\mathrm{T}_{\mathrm{c}}-$ control loop period

A method of minimizing above factor may be lengthening of control loop period but this may have negative impact on quality control. Other way is shortening transmission period but this is limited by number of nodes connected to the bus. By dividing 
network to many buses and spreading nodes among them we may make transmission period shorter (less nodes on single bus) but this enlarges cable harness. So there is a set of parameters to consider during system design.

Figure 1 presents time issues of using CAN-bus in control loop.

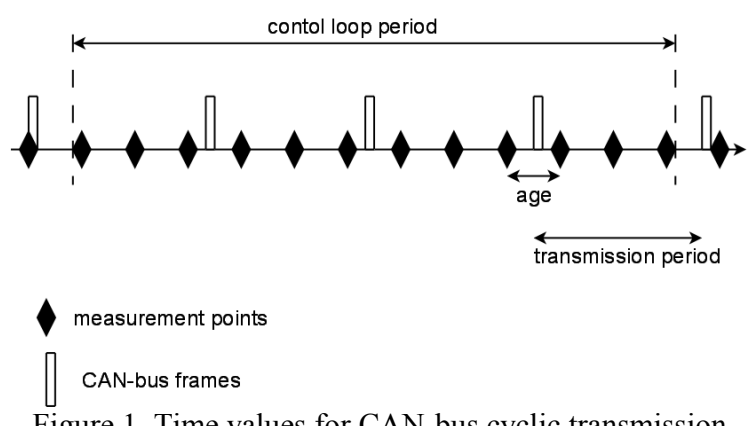

Figure 1. Time values for CAN-bus cyclic transmission

Another model of using CAN-bus is transmission on request. In that model gauges transmit their data only when they are asked by computer. Control station sends request for data to each node and they send their data in answer. Figure 2 presents time issues in such control.

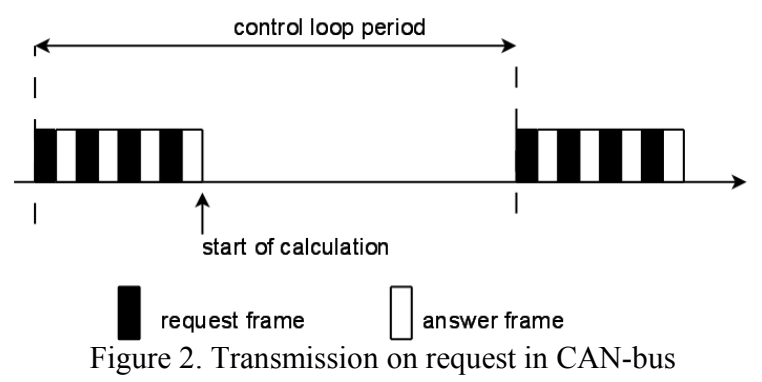

Asynchronous factor may be defined as follows:

$k_{a r}=\frac{n^{*} \max \left(t_{f}, t_{a}\right)+t_{f}}{T_{c}}$

where:

$\mathrm{k}_{\mathrm{ar}}$ - asynchronous factor for requested transmission

$t_{a}$ - maximum age of transmitted data

$t_{\mathrm{f}}$ - time of frame

$\mathrm{T}_{\mathrm{c}}$ - control loop period

$\mathrm{n}$ - number of answering nodes

\section{SIMULATOR}

Research on target environment is time consuming and expensive. Reconstructing the same environment for every experiment might be also problematic (e.g. preparing ground for excavating process). For this reason it might be very helpful to use simulator which imitates behavior of real machine altogether with data acquisition. That part should be placed in a computer system as replacement in order not to change rest of control system. Only at level of communication interface there should be possibility to switch between real machine and simulator. That kind of architecture keeps integrity of control algorithm (without need of implementing it in another environment) and upper layers of system (e.g. supervisory part) and keeps independency from mechanical part and communication subsystem (Figure 3).

The very first idea for using simulator was to test whether new control algorithm make sense and if that kind of control would work. For such kind of tests part imitating machinery would be very simplified. E.g. it would be based on assumption that velocity of rod is proportional to voltage of control signal. It seems that for such a simple test one could omit impact of signals delay, nonlinearities, direction of movement, change of forces in time (equipment position, weight, resistance of environment) crossdependencies between parts of equipment. More complex simulator in connection with verifications in real environment might be used to more complex research like impact of simplifying on quality of results or otherwise to test quality of model.

Next application of simulator might be checking how big is impact of test system parameters onto stability of control algorithm. One can check impact of resolution of gauges (e.g. what is the lower resolution which keeps system work correctly). Other test subjects are: frequency of measurements, asynchronous factor caused by CAN-bus. One more possibility is to test if system reacts properly in case of series problem like losing communication (suddenly broken gauges, etc.) 


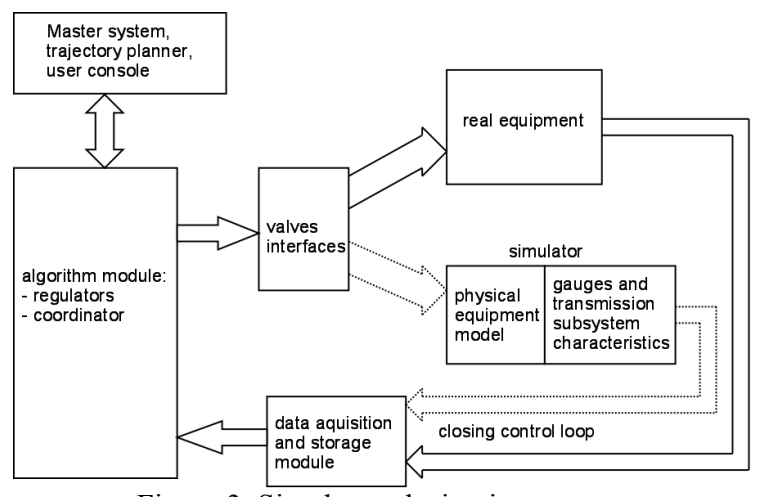

Figure 3. Simulator placing in a system

\section{VELOCITY VECTOR STABILISATION}

There are commonly known algorithms that makes machine to perform certain movements like PTP (point-to-point) algorithm and CP (continuous path) algorithm. Those algorithms are well described in wide range of publications in a field of automation and robotics. Our approach is based on velocity vector stabilization during movements towards points of path. Path is defined like in PTP but velocity of rods is monitored in way which allows reaching points in the same time by all rods. In case one rod is slowing down (e.g. environment resistance has changed and velocity regulator is not able to hold velocity or react immediately) other rods will slow down as well (Figure 4).

At the first glance this method looks like PTP algorithm with linear interpolation. The difference is that not positions of rods decide about output signals but their velocity. A drawback of this method is that one have to set up priorities for rods. It means that there is a relation of importance between them based on estimation "how hard job they do". E.g. there are two rods in a system: first one is moves in horizontal direction and second one vertically moves a part of construction/equipment. The second one (vertical) should have priority. Another way of choosing priority rod is on-line comparison of oil pressure in rods. Formulas for coordination calculation are shown below:

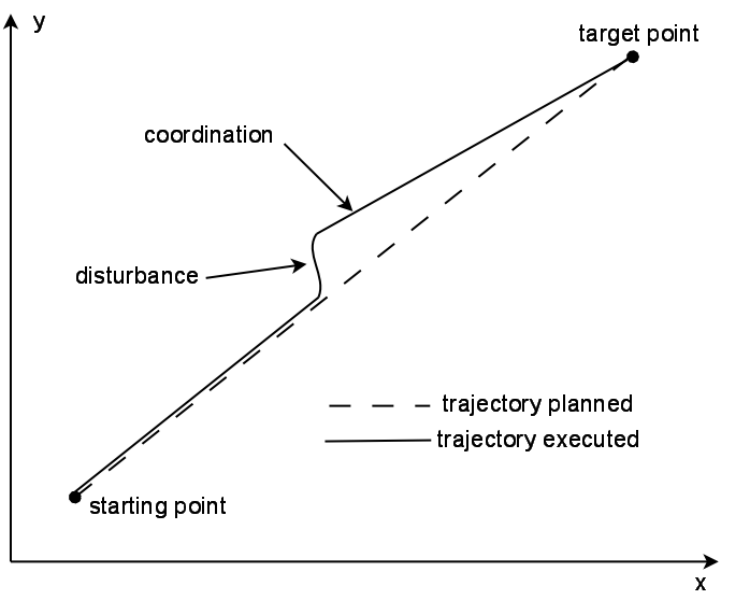

Figure 4. Idea of velocity vector stabilisation

$$
\begin{aligned}
& t=\frac{y-y_{t \arg e t}}{v_{y}} \\
& v_{x_{-} \text {new }}=\frac{x-x_{t \arg e t}}{t}
\end{aligned}
$$

where:

$\mathrm{y}$ - current position of priority rod

$\mathrm{y}_{\text {target }}-$ target position of priority rod

$\mathrm{v}_{\mathrm{y}}-$ current velocity of priority rod

$\mathrm{t}$ - estimated time when priority rod will reach target position $\mathrm{y}_{\text {target }}$

$\mathrm{X}$ - current position of other rod

$\mathrm{x}_{\text {target }}-$ target position of other rod

$\mathrm{v}_{\mathrm{x} \_ \text {new }}-$ new velocity for other rod

Quite important issue is fact that not all directions of movement are possible for excavator equipment, e.g. during excavating process when bucket is in a ground one can push beam up but is not possible to push it down so correction in normal direction to direction of movement may not be possible.

One of most important aims for this method is to not stop equipment during performing a task. Phases of accelerating and slowing down are difficult to manage (especially in context of rods coordination) and cause problems with quality so we wanted to minimize impact of those phases. Ideal situation is when velocity changes smoothly between segments of path without stopping in control points. The 
question is how to decide where to switch calculations onto next point. First attempt was switching when positions of rods reach target positions. This leads to overshoot because of inertia. This is presented on figure 5 .

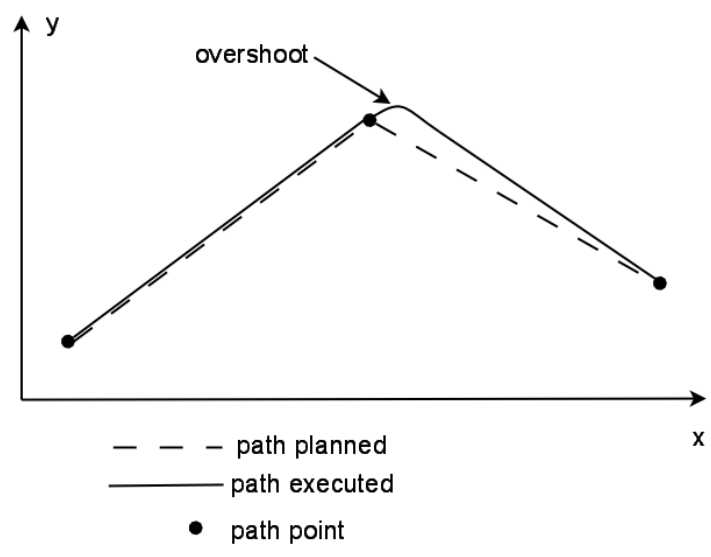

Figure 5. Switch after reaching point

Very important thing here is that width of surrounding should depend on inertia, velocity difference, shape of path (e.g. angle between path segments). When surrounding is too big there will be big shortcut as pictured on figure 6 .

Whilst in case of too small surrounding there will still be an overshoot. Like pictured on figure 7 .

One more feature should be mentioned. In case of high density path with wide surroundings there is a possibility to approximate round shapes by using set of lines. Figure 8 shows round approximation in Cartesian coordinate system. Otherwise it might be used to execute approximate lines in coordinate system of excavator.

Initial experiments show that idea of algorithm is correct but its efficiency, accuracy, behavior in border conditions, calculation of velocity regulator factors and optimization methods are subjects of further research.

\section{REFERENCES}

[1] "CAN Specification 2.0, Part A" from http://www.can-cia.org

[2] “CAN Specification 2.0, Part B" from http://www.can-cia.org

[3] Balluff transducers specification, BTL5-H120M0500-P-S92

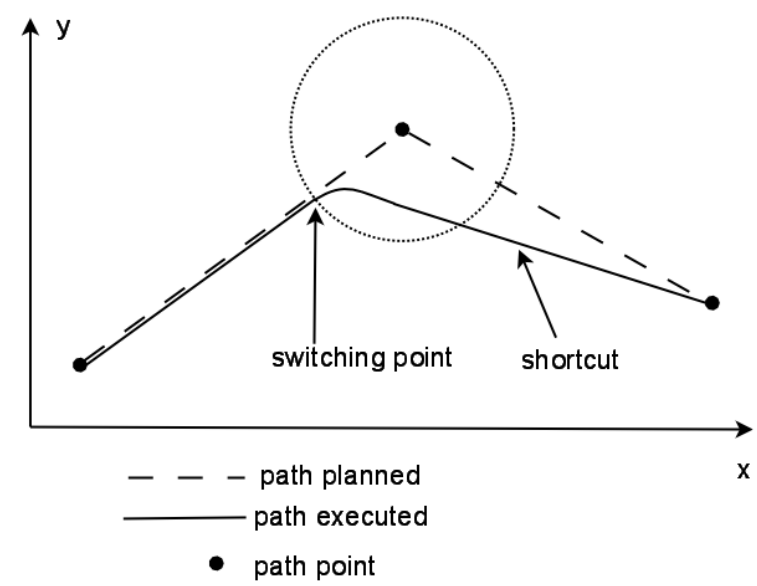

Figure 6. Switching in too big surrounding

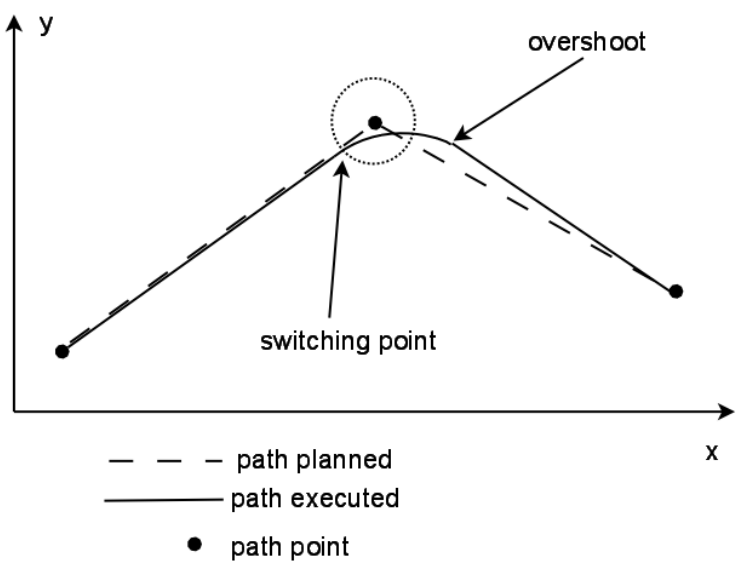

Figure 7. Switching in too small surrounding

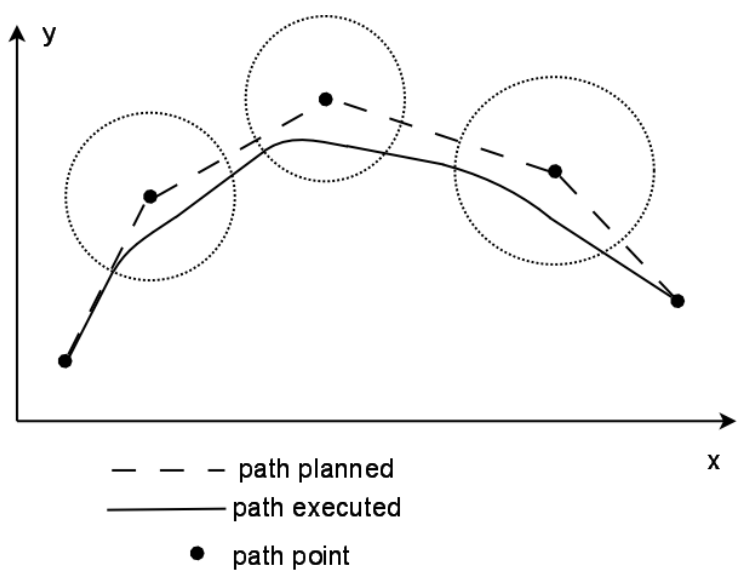

Figure 8 . High density path execution 